\title{
Radiant Heating Drying Method
}

National Cancer Institute

\section{Source}

National Cancer Institute. Radiant Heating Drying Method. NCI Thesaurus. Code C112982.

A process that removes water or volatile solvents from a bed of solids by relying on absorption of heat radiation by those solvents 